\title{
Factors Affecting Cesarean Section Rate Using Robson Classification: a 24-year-old retrospective analysis in a multiethnic population
}

\author{
Luigi Antonio De Vitis ${ }^{1}$, Stefano Manodoro ${ }^{2}$, Matteo Frigerio ${ }^{3}$, and Anna Maria Marconi ${ }^{1}$ \\ ${ }^{1}$ Università degli Studi di Milano \\ ${ }^{2}$ San Paolo University Hospital \\ ${ }^{3}$ San Gerardo Hospital
}

November 9, 2020

\begin{abstract}
Objective To evaluate factors affecting cesarean section (CS) rates in groups 1, 2A, 3, 4A, 5 and 10 of the "Ten Group Classification System" (TGCS). Design Retrospective analysis of deliveries occurred from January 1996 to December 2019. Setting A single hospital in Milan. Population Pregnant women belonging to groups 1, 2A, 3, 4A, 5 and 10 of the TGCS. Methods A binary logistic regression analysis was conducted. Included independent variables were maternal age, neonatal birthweight, immigrant status, use of obstetric analgesia, presence of diabetes, hypertension and obesity. Main outcome measures The effect of independent variables on CS rate was expressed as odds ratio. Results A total of 30591 deliveries were recorded. Advanced maternal age was an independent risk factor $(\mathrm{RF})$ in groups $1,2 \mathrm{~A}, 3$, and $4 \mathrm{~A}$; diabetes was a risk factor in groups 1 and 5; obesity was a $\mathrm{RF}$ in groups 1 and $2 \mathrm{~A}$ and a protective one in group 5; hypertension was a $\mathrm{RF}$ in groups $2 \mathrm{~A}, 5$ and 10 ; macrosomia was a RF in groups $1,2 \mathrm{~A}$ and 3 ; use of obstetric analgesia was either a $\mathrm{RF}$ in group 1 , and a protective factor in groups 2A, 5 and 10; immigrant status was either a protective factor in groups 1 and 10, and a RF in group 4A. Conclusion The TGCS is a well-established method to compare CS rates between institutions; however, inside each group, many factors can influence the CS rate and they have to be taken into consideration when comparing CS rates.
\end{abstract}

\section{Title}

Factors Affecting Caesarean Section Rate Using Robson Classification: a 24-year-old retrospective analysis in a multiethnic population

\section{Authors}

Luigi Antonio De Vitis $^{1,3^{*}}$, Stefano Manodoro ${ }^{1 *}$, Matteo Frigerio ${ }^{2}$, Anna Maria Marconi ${ }^{1,3}$

*LADV and SM equally contributed to this study

\section{Affiliations}

${ }^{1}$ Unit of Obstetrics and Gynaecology, San Paolo Hospital Medical School, ASST Santi Paolo e Carlo, Milano, Italy

${ }^{2}$ Unit of Obstetrics and Gynaecology, San Gerardo University Hospital, Monza, Italy

${ }^{3}$ Department of Health Sciences, University of Milano, Italy

\section{Corresponding Author}


Stefano Manodoro, email:stefano.manodoro@gmail.com, Unit of Obstetrics and Gynaecology, San Paolo Hospital Medical School, ASST Santi Paolo e Carlo, via Antonio di Rudinì 8, 20142, Milano, Italy, 0281844419

\section{Running title}

Factors affecting CS rates and the TGCS

\section{Abstract}

\section{Objective}

To evaluate factors affecting caesarean section (CS) rates in groups 1, 2A, 3, 4A, 5 and 10 of the "Ten Group Classification System" (TGCS).

\section{Design}

Retrospective analysis of deliveries occurred from January 1996 to December 2019.

\section{Setting}

A single hospital in Milan.

\section{Population}

Pregnant women belonging to groups 1, 2A, 3, 4A, 5 and 10 of the TGCS.

\section{Methods}

A binary logistic regression analysis was conducted. Included independent variables were maternal age, neonatal birthweight, immigrant status, use of obstetric analgesia, presence of diabetes, hypertension and obesity.

\section{Main outcome measures}

The effect of independent variables on CS rate was expressed as odds ratio.

\section{Results}

A total of 30591 deliveries were recorded. Advanced maternal age was an independent risk factor (RF) in groups 1,2A, 3, and 4A; diabetes was a RF in groups 1 and 5; obesity was a RF in groups 1 and $2 \mathrm{~A}$ and a protective one in group 5; hypertension was a $\mathrm{RF}$ in groups $2 \mathrm{~A}, 5$ and 10 ; macrosomia was a $\mathrm{RF}$ in groups $1,2 \mathrm{~A}$ and 3 ; use of obstetric analgesia was either a $\mathrm{RF}$ in group 1, and a protective factor in groups $2 \mathrm{~A}, 5$ and 10; immigrant status was either a protective factor in groups 1 and 10, and a $\mathrm{RF}$ in group $4 \mathrm{~A}$.

\section{Conclusion}

The TGCS is a well-established method to compare CS rates between institutions; however, inside each group, many factors can influence the CS rate and they have to be taken into consideration when comparing CS rates.

\section{Tweetable abstract}

Obstetrics and maternal-fetal factors increase or reduce CS rate in a different way depending on Robson group.

Keywords : Robson's group; TGCS; ten group classification system; caesarean section; caesarean section rate

\section{Introduction}

Since $1985^{1}$, the World Health Organization (WHO) has considered the ideal caesarean section (CS) rate to be between $10 \%$ and $15 \%$ with the crude rate of CS proposed as a global indicator to evaluate the quality of obstetric care. However, since then, CS rates have gradually increased, raising concerns on the potential 
negative effects on mother and infant health, and many efforts have been made to reduce the number of unnecessary interventions. In 2001 Robson $^{2}$ proposed the "Ten Group Classification System" (TGCS), a totally inclusive and mutually exclusive classification system that divides the obstetric population into 10 groups with the aim of comparing caesarean section rates over time in one single unit and among different units, to improve perinatal care. In $2017^{3}$ the WHO has endorsed the TGCS, proposing that it should be considered "a global standard for assessing, monitoring and comparing CS rates within healthcare facilities over time, and between facilities". A relatively recent review ${ }^{4}$ of the literature that included 73 papers on the use of the Robson classification in more than 33 million women in 31 countries showed that, despite its valuable utility, among the limitations reported by users, there was a failure to take into account the indication to the CS and the characteristics of both the mother and the fetus, which can significantly influence the CS rate (e.g. maternal age or fetal growth, to name just a few). Consequently, it is possible to find different CS rates in the same Robson group, in relation to different countries, but also in the same institution, in relation to different types of women. These differences may influence the comparison among hospitals or inside the same hospital if the population characteristics change. Maternal age, immigrant status, body mass index and diseases (for instance, gestational diabetes mellitus or hypertension), or neonatal birthweight and the use of obstetric analgesia are not taken into consideration by the TGCS but they potentially influence the CS rate. Our hypothesis is that these factors may influence the probability of giving birth by caesarean section, within each individual Robson group.

Therefore, the purpose of our study was to evaluate which obstetric and maternal-fetal variables affect the CS rate within the individual Robson groups and to verify any differences between the groups. As secondary results we analysed the time course of the CS rate for the Robson groups over a period of 24 years.

\section{Methods}

The current study was conducted at the Unit of Obstetrics and Gynaecology of the San Paolo Hospital, Medical School in Milano, Italy. The Unit is a secondary referral centre, i.e. comprises a maternal intensive care unit (ICU) but not neonatal ICU. The audit system in our institution involves a weekly discussion of relevant clinical cases, the use of standardized and up-to-date protocols and the publication of an annual report about labour, delivery, maternal and neonatal outcomes of all women. The majority of the nonItalian women delivering in our Unit, are assisted by a non-profit social cooperative that deals with cultural mediation. Data were obtained from an electronic database that includes information on deliveries that occurred in our clinic from 1996 to 2019. Data were already available for the analysis for all women as part of the annual clinical report of the Unit. Obstetric outcomes, maternal and fetal characteristics included in the database are collected from medical records at the end of each month by residents in Obstetrics and Gynaecology. The database includes all the live births and stillbirths from 23 weeks of gestational age. The board of the San Paolo Department of Health Sciences approved the study protocol. When information on parity, number of fetuses, previous caesarean section, onset of labour, gestational age, and fetal presentation were all available, women were classified according to the modified TGCS; for the purpose of the analysis we selected women belonging to Group 1 (nulliparous, single cephalic, [?]37 weeks, in spontaneous labour), 2A (nulliparous, single cephalic, [?]37 weeks, induced), 3 (multiparous, excluding previous CS, single cephalic, [?]37 weeks, in spontaneous labour, 4A (multiparous, excluding previous CS, single cephalic, [?]37 weeks, induced) and 10 (all single cephalic, [?]36 weeks). We also included in the regression analysis women of Group 5 (previous CS, single cephalic, [?]37 weeks) only if they attempted a trial of labour after caesarean (TOLAC). Hereafter we will refer to these women as Group 5. We excluded from the analysis women of Groups $2 \mathrm{~B}$ (nulliparous, single cephalic, [?]37 weeks, CS before labour), 4B (multiparous, excluding previous CS, single cephalic, [?]37 weeks, CS before labour), 6 (all nulliparous breeches), 7 (all multiparous breeches), and 9 (all abnormal presentations) because they underwent elective caesarean sections as for protocol. We also excluded multiple pregnancies (group 8) due to the presence of several potentially confounding factors and to the limited population The analysis was conducted inside each group and not between groups. Induction of labour was defined as the use of any mechanical method (intrauterine Foley catheter or Cervical Ripening Balloon), medication (prostaglandins or oxytocin) or amniotomy when not in labour. 
The CS rate was calculated for each group as the number of CS out of the number of all the deliveries. We also calculated the CS rate per year in each group. The impact of advanced age (maternal age equal to or higher than 40 year), diabetes (including pre-pregnancy diabetes mellitus and gestational diabetes mellitus), hypertensive disease (including pregestational hypertension, gestational hypertension, preeclampsia or eclampsia), fetal macrosomia (neonatal birthweight higher than $4000 \mathrm{~g}$ ), obesity (pre-pregnancy body mass index [?]30 kg $/ \mathrm{m}^{2}$ ), obstetric analgesia and immigrant status was evaluated. Gestational diabetes mellitus and hypertensive disease of pregnancy were diagnosed according to the guidelines available at the time of the pregnancy. The dependent variable was the type of delivery, i.e. vaginal (both spontaneous or operative) or caesarean section. The variable macrosomia was not included in the analysis of group 10 because all the newborn weighed less than $4000 \mathrm{~g}$. A binomial logistic regression was performed to ascertain the effects of variables on CS rates and odds ratio were calculated. For missing values, listwise deletion approach was applied. Linear regressions and Anova tests were performed to evaluate temporal trends in CS rates for considered Robson groups.

All calculations were performed using SPSS version 25.0 (SPSS Inc, Chicago, IL, USA). A p-value lower than 0.05 was considered statistically significant.

\section{Results}

In our Unit, in the period from 1996 to 2019 there were 42016 births. In 32360 women (77\%) all the variables needed for the TGCS were recorded: 1769 (5.5\%) women belonged to Groups 2B, 4B, 6, 7, 8 and 9 and were therefore excluded from analysis. Thus, a total of 30591 deliveries are included in our analysis: 9236 in Group 1 (28.5\%); $4717(14.6 \%)$ in Group 2A; $8784(27.1 \%)$ in Group 3; 2560 (7.9\%) in Group 4A; $1847(5.7 \%)$ in Group 10 and $3447(10.7 \%)$ in Group 5. In this latter Group, 887 women (25.7\%) attempted TOLAC. Maternal characteristics and type of delivery related to the entire population according to Robson Groups are listed in Table 1. Among immigrants 36.2\% were from Africa, 23.3\% from Eastern Europe, 22.0\% from Asia, 18.0\% from South-America. In our institution from 1996 to 2019 the CS rate was stable in groups $1(6.2+-2.0 \%), 3(1.8+-0.7 \%), 4 \mathrm{~A}(4.1+-2.2 \%)$, while it decreased in groups $2 \mathrm{~A}$ (from $27.9 \%$ in 2000 to $19.9 \%$ in $2019, \mathrm{~F}=0.03$ ), 5 (from $94.2 \%$ in 1998 to $75.3 \%$ in $2019, \mathrm{~F}<0.0001$ ) and 10 (from $58.9 \%$ in 2000 to $28.3 \%$ in 2019, $\mathrm{F}<0.0001$ ) (Figure S1). Perinatal mortality (i.e. the number of stillbirths from 28 weeks and deaths in the first week of life) was 3(97 cases) and it remained stable throughout the study period.

Table 2 reports the results of the binomial logistic regression, including odds ratio (OR), $95 \%$ confidence interval (CI) and p-value.

The age of the woman greater than or equal to 40 appears to be a risk factor (RF) for CS in all groups except 5 and 10. The presence of pregestational-gestational diabetes is a RF for CS only in Groups 1 and 5. Not surprisingly, gestational hypertension is a RF for CS in preterm pregnancies (Group 10) and in nulliparous induced (Group 2A), but also in Group 5. Obesity represents a RF for CS in nulliparous (Groups 1 and 2A) whilst is protective in Group 5. The presence of a neonatal weight $>4000$ is a $\mathrm{RF}$ for CS in nulliparous (Group 1), especially when induced (Group 2A), and multiparous in spontaneous labour (Group 3). Obstetric analgesia is protective against CS in nulliparous induced (Group 2A), women in TOL (Group 5) and preterm (Group 10).

Being immigrant was a RF for CS in multiparous induced (Group 4A) while it was protective in nulliparous in spontaneous labour (Group 1) and preterm (Group 10).

\section{Discussion}

\section{Main findings}

Rates of CS are rising worldwide, but determinants of this increase remain controversial. In particular, despite the utility of TGCS in allowing standardized comparisons thus helping to define strategies to reduce CS rates, it does not account for several maternal and fetal clinical variables that can potentially affect the mode of delivery ${ }^{4}$. For instance, advanced maternal age, obesity, macrosomia, diabetes and hypertension are well-known RFs for $\mathrm{CS}^{5-11}$. Others, like epidural analgesia and immigrant status have a more controversial 
relationship ${ }^{12,13}$. In this study we aimed to estimate the impact of obstetric and maternal-fetal variables in affecting CS rate in different Robson groups and to analyse the trends of CS rates in the given period in our Unit. We found that all the considered variables (advanced maternal age, diabetes, hypertension, fetal macrosomia, obesity, epidural analgesia, and immigrant status) altered the CS rate at least in one Robson group. Nevertheless, they differently influenced the CS rate in relation to the Robson groups. Moreover, we found that the rate of CS in the last 24 years decreased significantly in Robson groups $2 \mathrm{~A}, 5$ and 10.

\section{Interpretation}

Advanced maternal age resulted as an independent RF for caesarean section in Robson groups 1, 2A, 3, and $4 \mathrm{~A}$. We have recently shown ${ }^{11}$ that maternal age [?]40 years represents a $\mathrm{RF}$ on $\mathrm{CS}$ rate strongest than parity.

The presence of diabetes significantly increased the risk of CS delivery in groups 1 and 5 but its effect was not significant in the other groups. Our data are in contrast with the results of Zeki et al ${ }^{14}$ that showed that diabetes increases the CS rate in all groups except Group 1. These differences may be due to the different CS rate in the study populations (i.e. lower in our study). Despite the uncertainty of these evidences, diabetes has to be taken into consideration when comparing CS rates, since it is likely to play a role regardless of the Robson group.

Hypertensive disease was identified as a RF in groups 2A (OR 1.32) and 5 (OR 3.49), probably because the decision to perform a CS in labour is taken earlier if a nulliparous undergoes induction of labour for hypertension or she has a previous uterine scar. Also, hypertension represented the strongest RF for CS in group 10 (OR 3.7) probably because in this group the disease is more severe thus to anticipate the time of delivery is a common prudential clinical behaviour. In multiparous without a uterine scar, hypertension did not reach statistical significance. The association between severity of hypertension and CS has been previously shown ${ }^{6}$. However, in our study, similarly to what Gerli et al. ${ }^{15}$ showed in Robson's classes 1 and 3, hypertension represents a RF for caesarean section in nulliparous but not in multiparous. Obesity increased the CS rates in nulliparous women at term (i.e. groups 1 and $2 \mathrm{~A}$ ) but not in multiparous at term (i.e. groups 3 and $4 \mathrm{~A}$ ). Also, in this case our results are similar to those of Gerli et al. ${ }^{15}$ in a population similar to ours. However, unexpectedly, in our population, obesity decreased the risk of CS in group 5 women attempting TOLAC. It is well known that increasing BMI has an inverse association with the likelihood of achieving vaginal birth ${ }^{16}$. A possible explanation could be that, given that caesarean sections in labour and in obese women, involves greater difficulties and complications, the decision is postponed, in an attempt to avoid surgery, with a consequent greater probability of obtaining a vaginal birth ${ }^{16}$; alternatively, it could be that only obese women who are more likely to be successful in vaginal delivery are admitted to TOL. Nulliparous women at term (group 1 and 2A) and multiparous women in spontaneous labour (group 3) with macrosomic fetuses were more likely to have a CS. Of note, macrosomia was one of the two RFs that more than doubled the risk of CS in group 3. The association between macrosomia and CS is well-known ${ }^{9}$ and a recent trial ${ }^{10}$ proved that, when macrosomia is suspected, labour induction at an earlier gestational age increases the likelihood of vaginal delivery.

Obstetrics analgesia and ethnicity behaved as risk or protective factors depending on the Robson group. According to our data, obstetric analgesia increased the risk of CS in nulliparous women in spontaneous labour, while decreased the risk when labour was induced. In addition, it decreased the risk of CS in groups 5 and 10. A recent meta-analysis ${ }^{13}$ concluded that epidural analgesia has no impact on the risk of caesarean section. As a matter of fact, in our analysis we included every type of pharmacological pain control (i.e. intravenous drugs, epidural analgesia), so that it is difficult to make any comparison. Probably, more studies are necessary to define the role of obstetric analgesia as a risk or protective factor for CS rates in the different Robson groups.

Being immigrant, in our study, was a protective factor in groups 1 and 10 and a RF in group 4A. A metanalysis by Merry et al. ${ }^{17}$ that evaluated CS rates between immigrants and non-immigrants women revealed higher CS rate for Sub-Saharan African, Somali and South Asian women; higher emergency rates 
for North African/West Asian and Latin American women; and lower rates for Eastern European and Vietnamese women. However, they did not evaluated differences between Robson groups. A study by Minsart et al ${ }^{12}$ demonstrated an increased risk in group 1, 2, 3 and 4 for mothers from Sub-Saharan Africa compared with Belgian natives, while a reduced risk for East European women in group 1. A recent study by Linard et al ${ }^{18}$ demonstrated a higher CS rate for Sub-Saharan African women in group 5 with only one previous uterine scar, compared to French women. Anyway, it is not possible to compare these studies because the ethnic groups taken into consideration are different and data are heterogeneous.

The statistically significant reduction of CS rate in Robson groups $2 \mathrm{~A}, 5$, and 10 reflects the efforts made by our institution to avoid unnecessary CS. It is likely that the weekly audit system adopted in our institution, together with the use of standardized and updated protocols and the publication of annual reports, has played, and can continue to play, a decisive role in reducing CS rates. The implementation of the "trial of labour after caesarean section" helped to reduce the number of CS in Robson group 5, while the reduction in group $2 \mathrm{~A}$ it is probably due to the fact that our labour induction protocol allows wait a longer period of time to declare the procedure failed ${ }^{19}$. On the other hand, we think that our CS rate in multiparous women (Robson groups 3 and 4A) and in nulliparous women in spontaneous labour was already adequate, according to international standards ${ }^{2}$, so that a further reduction could be harmful.

\section{Strengths and Limitations}

The main limitation of our study is that it is a retrospective study, articulated in 24 years, in which the standards of care, the characteristics of the women and the protocols have obviously changed, therefore the decision to perform a caesarean section in group 2A 4A may have been different in 2000 or 2019. Moreover, neonatal ICU is not available, so that group 10 mainly included women after the 34 weeks of gestational age.

However, these can also be considered the major strength, in the sense that the changes that have taken place over the years have been counterbalanced by the uniformity of behaviour, in the same institution.

\section{Conclusion}

Obstetrics and maternal-fetal factors should also be considered when comparing CS rates in Robson's groups. In our study, the woman's age $>40$ years, diabetes, hypertension, fetal macrosomia, obesity, obstetric analgesia, and immigrant status were identified as risk or protective factors with different impact depending on the Robson group. In comparing the CS rate between different institutions, therefore, the meaning of the attribution to the different groups should not disregard the characteristics of the population included.

Acknowledgments : none.

Disclosure of interests : all authors report no conflicts of interests related to this manuscript.

Contribution to authorship : LADV, SM contributed to data acquisition, study design, data analysis, data interpretation and manuscript writing; MF contributed to data analysis, data interpretation and manuscript writing and revision; AMM contributed to study design, data interpretation, manuscript writing and critical manuscript revision. All authors agree with the final version, and agree to be accountable to the integrity of the data published.

Details of ethics approval : this study was approved by the board of the San Paolo Department of Health Sciences.

\section{Source of funding : none.}

\section{References}

1. WHO. Appropriate technology for birth. Lancet. 1985 Aug 24;2(8452):436-7.

2. Robson MS. Can we reduce the caesarean section rate? Best Pract Res Clin Obstet Gynaecol. 2001 Feb;15(1):179-94.

3. WHO. Robson Classification: Implementation Manual. Geneva; 2017. 
4. Betran AP, Vindevoghel N, Souza JP, Gulmezoglu AM, Torloni MR. A systematic review of the Robson classification for caesarean section: what works, doesn't work and how to improve it. PLoS One. 2014;9(6):e97769.

5. Favilli A, Pericoli S, Acanfora MM, Bini V, Di Renzo GC, Gerli S. Pregnancy outcome in women aged 40 years or more. J Matern Fetal Neonatal Med. 2012 Aug;25(8):1260-3.

6. Yucesoy G, Ozkan S, Bodur H, Tan T, Caliskan E, Vural B, et al. Maternal and perinatal outcome in pregnancies complicated with hypertensive disorder of pregnancy: a seven year experience of a tertiary care center. Arch Gynecol Obstet. 2005 Nov;273(1):43-9.

7. Ovesen PG, Jensen DM, Damm P, Rasmussen S, Kesmodel US. Maternal and neonatal outcomes in pregnancies complicated by gestational diabetes. a nation-wide study. J Matern Fetal Neonatal Med. 2015;28(14):1720-4.

8. Calderon AC, Quintana SM, Marcolin AC, Berezowski AT, Brito LG, Duarte G, et al. Obesity and pregnancy: a transversal study from a low-risk maternity. BMC Pregnancy Childbirth. 2014 Jul 28;14:249.

9. Vidarsdottir H, Geirsson RT, Hardardottir H, Valdimarsdottir U, Dagbjartsson A. Obstetric and neonatal risks among extremely macrosomic babies and their mothers. Am J Obstet Gynecol. 2011 May;204(5):423.e16 .

10. Boulvain M, Senat MV, Perrotin F, Winer N, Beucher G, Subtil D, et al. Induction of labour versus expectant management for large-for-date fetuses: a randomised controlled trial. Lancet. 2015 Jun 27;385(9987):2600-5.

11. Marconi AM, Manodoro S, Cipriani S, Parazzini F. Cesarean section rate is a matter of maternal age or parity? J Matern Fetal Neonatal Med. 2020 Aug 12:1-4.

12. Minsart AF, De Spiegelaere M, Englert Y, Buekens P. Classification of cesarean sections among immigrants in Belgium. Acta Obstet Gynecol Scand. 2013 Feb;92(2):204-9.

13. Anim-Somuah M, Smyth RM, Cyna AM, Cuthbert A. Epidural versus non-epidural or no analgesia for pain management in labour. Cochrane Database Syst Rev. 2018 May 21;5(5):Cd000331.

14. Zeki R, Oats JJN, Wang AY, Li Z, Homer CSE, Sullivan EA. Cesarean section and diabetes during pregnancy: An NSW population study using the Robson classification. J Obstet Gynaecol Res. 2018 May;44(5):890-8.

15. Gerli S, Favilli A, Franchini D, De Giorgi M, Casucci P, Parazzini F. Is the Robson's classification system burdened by obstetric pathologies, maternal characteristics and assistential levels in comparing hospitals cesarean rates? A regional analysis of class 1 and 3. J Matern Fetal Neonatal Med. 2018 Jan;31(2):173-7.

16. ACOG. ACOG Practice Bulletin No. 205: Vaginal Birth After Cesarean Delivery. Obstet Gynecol. 2019 Feb;133(2):e110-e27.

17. Merry L, Small R, Blondel B, Gagnon AJ. International migration and caesarean birth: a systematic review and meta-analysis. BMC Pregnancy Childbirth. 2013 Jan 30;13:27.

18. Linard M, Deneux-Tharaux C, Luton D, Schmitz T, Mandelbrot L, Estellat C, et al. Differential rates of cesarean delivery by maternal geographical origin: a cohort study in France. BMC Pregnancy Childbirth. 2019 Jun 27;19(1):217.

19. Caughey AB, Cahill AG, Guise JM, Rouse DJ. Safe prevention of the primary cesarean delivery. Am J Obstet Gynecol. 2014 Mar;210(3):179-93.

Table 1. Maternal characteristics and type of delivery of both the entire population and divided according to Robson's groups.

Table 2. Binary logistic regression analysis results. 
Figure S1. Supplementary material. Caesarean section rate from 1996 to 2019.

\section{Hosted file}

Table 1.pdf available at https://authorea.com/users/374142/articles/491742-factors-affectingcesarean-section-rate-using-robson-classification-a-24-year-old-retrospective-analysisin-a-multiethnic-population

\section{Hosted file}

Table 2.pdf available at https://authorea.com/users/374142/articles/491742-factors-affectingcesarean-section-rate-using-robson-classification-a-24-year-old-retrospective-analysisin-a-multiethnic-population 